einigung dualer Seinsweisen im Objekt hängt es vom Beobachter $a b$, in welcher Gestalt ihm das Objekt entgegentritt. Die quantenmechanische Statistik ist also nicht nur wegen unseres beschränkten Wissens subjektgefärbt, sondern auch wegen der Gleichwertigkeit verschiedener $G e$ stalten zur Beschreibung des Seins. Dabei ist zu beachten, daß die Behauptung der Gleichwertigkeit der beiden Gestalten: Korpuskel und Welle, eine objektive Aussage darstellt, die aus der speziellen Art der Korrelation, nämlich der Kom- plementarität, folgt. Die Heis en bergschen Gedankenexperimente beweisen in diesem Zusammenhang, daß man sich der objektiv bestehenden Bindung durch die Komplementarität auf keine Weise entziehen kann. Sie geben uns ebenso von der Komplementarität Kunde wie z. B. die Stabilität der Elektronenbahnen in unberührten Wasserstoffatomen.

Die vorstehenden Untersuchungen sind dureh Diskussionen über v. We i z sä ckers oben zitierte ${ }^{15}$ Vorträge mit Hrn. Prof. Fues angeregt, für die ich auch an dieser Stelle herzlich danken möchte.

\title{
Zur Theorie der Neuen Sterne
}

\author{
Von Gerhard Miczaika \\ Aus der Badischen Landessternwarte Heidelberg-Königstuhl \\ (Z. Naturforschg. 2 a, 216-219 [1947]; eingegangen am 30. September 1946)
}

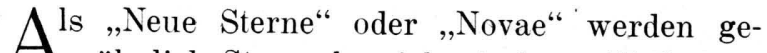
Awöhnlich Sterne bezeichnet, deren Helligkeit sehr plötzlich, etwa in einigen Stunden oder ein bis zwei Tagen, um mehrere Größenklassen ansteigt, um dann erheblich langsamer wieder zum Ausgangswert, meist unter sekundären Schwankungen, zurückzukehren. Aus den spektroskopischen Befunden geht hervor, daß der Ausbruch mit einer gewaltigen Vergrößerung der Sternoberfläche verbunden ist, die im wesentlichen als eine Vergrößerung der Atmosphäre des Sterns aufzufassen sein dürfte. Violettverschiebung der Absorptionslinien, Verbreiterung der Emissionslinien und schließlich Auftreten typischer Nebellinien sind die wichtigsten Charakteristika für das Ablaufen des Novaprozesses. Zur Kennzeichnung einer Nova müssen beide Kriterien, plötzlicher Helligkeitsanstieg und spektrale Merkmale, nachweisbar sein.

Die bei einem Ausbruch abgegebene Energie ist nur ein geringer Bruchteil des potentiellen Energievorrats eines Sterns, der der Größenordnung nach etwa $10^{49} \mathrm{erg}$ beträgt. B i e r m a n $n^{1}$ schätzt die bei einem Ausbruch abgegebene Energie aus der Lichtkurve auf etwa $10^{44} \mathrm{erg}$. Massenmäßig dürfte ebenfalls nur ein kleiner Teil des Sterns an der Aufblähung und Ausbildung einer ausgedehnten Atmosphärenschicht teilnehmen.
\end{abstract}

1 L. B i erm a n n, Z. Astrophysik 18, 344 [1939].
Das eindrucksvolle Phänomen der Novae ist häufig und bereits sehr früh Gegenstand von Hypothesen über seinen Ursprung gewesen, ohne daß bisher eine befriedigende Erklärung gefunden worden wäre. Die zur Zeit zweifellos plausibelsten Vorstellungen haben Uns öld ${ }^{2}$ und später B i e r m a $n n^{1}$ entwickelt.

Uns öld weist darauf hin, daß in einer Sternatmosphäre eine herabsinkende Gasmasse nicht nur ihren Druck und ihre Temperatur, sondern auch ihren Ionisationsgrad ändert. Bei Temperaturerhöhung würde also die Translationsenergie der Teilchen vergrößert und Ionisationsarbeit geleistet. Das Verhalten des Gases nähert sich dem eines mehratomigen, da das effektive Verhältnis der spezifischen Wärmen $\bar{x}<5 / 3$ wird. Unter der Voraussetzung, daß eine Schicht hauptsächlich aus Wasserstoff aufgebaut ist, und Annahme plausibler Werte für Temperatur und Massenabsorptionskoeffizient sinkt $\bar{x}$ unter Umständen sogar unter ${ }^{4} / 3$. Dies bedeutet bekanntlich instabiles Strahlungsgleichgewicht und thermodynamische Stabilität der ausgleichenden Konvektionsströme. Un söld glaubt nun, daß die Instabilität auch anders zum Ausgleich kommen kann. Der thermodynamisch instabile Zustand eines Teils des Sterns bedeutet noch nicht dynamische Instabilität des Ganzen. Eine kleine Störung jedoch

2 A. Unsöld, Z. Astrophysik 1, 138 [1930]. 
genügt, um die labile Zone zum Umklappen zu bringen, wodurch sehr heiße Teile des Sterns nach außen kommen und die Photosphäre fortgetrieben wird. Ein zweimaliger Ausbruch desselben Sterns wäre als Durchlaufen einer größeren Zone durch die $L$ - oder $M$-Ionisation mit Umklappen der entstehenden Instabilitätszone aufzufassen.

Ähnliche Vorstellungen entwickelt Bi ermann. Die potentiellen Novae sind Sterne frühen Spektraltyps mit $M_{v i s}=+4^{m}$, die in unregelmäßigen Intervallen von 100 bis 10000 Jahren aufleuchten und danach wieder in den alten Zustand zurückkehren. Ihre Energiequelle ist die Kontraktion, da sie ihren Wasserstoff durch Kernprozesse fast vollständig verloren haben. Für die Energie des Ausbruchs steht nach Biermann nur potentielle Energie einer Instabilität der Schichtung und Ionisationsenergie zur Verfügung, diese allerdings auch nur in Instabilitätszonen, da sie sich bei stabiler Schichtung nicht mehr mit anderen Energieformen umsetzt.

Biermann zeigt, daß unter der Voraussetzung sehr geringen Wasserstoffgehaltes und Heliumgehaltes von $<10 \%$ der äußeren Schichten bei Temperaturen bis zu $10^{7}{ }^{\circ}$ Instabilität der Schichtung möglich ist. Solange Entartung keine Rolle spielt, verläuft die Kontraktion möglicherweise nicht homolog. Es können ausgedehnte Zonen im Sterninnern an der Grenze der thermischen Stabilität entstehen, die gelegentlich zusammenbrechen. Die Plötzlichkeit des Vorganges wird durch die hohe Schallgeschwindigkeit (316 km/sec nach Bi e rma n n) verständlich.

Der Stern, der bereits vorher infolge Erlöschens der atomaren Energieproduktion die Hauptreihe verlassen hatte und im Gebiet zwischen Hauptreihe und dem der weißen $Z$ werge steht, wird sich also weiter kontrahieren und schließlich als weißer Zwerg enden.

Die sekundäre Veränderlichkeit der Novae im „Ruhezustand“ spricht vielleicht für Konvektion in den äußeren Schichten, so daß hierin ein Argument gegen die Vorstellung vom Aufbau einer instabilen Zone erblickt werden kann.

Eddington ${ }^{3}$ macht die erstaunliche Feststellung, daß es, nach ihrem Wasserstoffgehalt zu urteilen, offenbar zwei Gruppen von weißen Zwergen gibt. Vertreter der wasserstoffreichen

3 A. S. Eddington, Monthly Notices Roy. astronom. Soc. 99, 595 [1939].
Gruppe ist Sirius B (68\%), in die wasserstoffarme Gruppe gehört u. a. van Maanen Nr. 2 (5 bis 11\%). Eddingt on diskutiert die Möglichkeit, daß am Anfang und am Ende der Sternentwicklung auf der Hauptreihe weiße Zwerge stehen. Der Beginn eines Sterns ist in dieser Vorstellung eine Wasserstoffkugel ohne subatomare Energieerzeugung, die ihren Energiebedarf also allein aus der Kontraktion deckt. Wenn die Dichte die Größenordnung derjenigen der weißen Zwerge erreicht hat, beginnt der Aufbau der schweren Elemente. Nach Bildung der notwendigen Katalysatoren setzt Energieerzeugung aus Kernreaktionen ein, die den - Energiebedarf der Abstrahlung übersteigt, so daß der Stern expandiert. Schließlich kommt der Aufbau der höheren Elemente zum Stillstand und die CN-Kette bleibt die einzige Energiequelle. Der große Betrag an Energie aus der Wasserstoffumwandlung führt vielleicht zu einer Katastrophe bei der Expansion, bevor der Stern den Gleichgewichtszustand auf der Hauptreihe erreicht. Der Stern kehrt nach Erschöpfung seines Wasserstoffvorrates wieder in den Zustand der weißen Zwerge zurück.

Diese Hypothese hat kaum Konsequenzen, die zur Zeit an der Erfahrung in bezug auf die Novae prüfbar sind. Gegen sie scheint zu sprechen, daß ein Nova-Ausbruch einen Stern nicht wesentlich $\mathrm{zu}$ verändern scheint, während ein so stark wasserstoffhaltiges Gebilde, wie es die weißen Zwerge der primären Phase sind, in einer den ganzen Stern erfassenden Explosion umgebaut werden würde. Sie schließt auch die Möglichkeit wiederholter Ausbrüche aus.

Ein sehr wesentlicher Zug des Novaphänomens scheint die Wiederholungstendenz zu sein. Tatsächlich ist gerade in den letztèn Jahren eine größere Anzahl zweiter und dritter Ausbrüche älterer Novae beobachtet worden ${ }^{4}$, worunter vor allem T Pyxidis, RS Ophiuchi, $U$ Scorpii, $T$ Coronae borealis, Nova Sagittae Nr. 2 zu nennen sind. Die Ausbrüche sprechen ohne $Z_{w}$ eifel für èchte Novae. Schließlich ist in diesem Zusammenhang auch auf die den Novae vermutlich verwandten SS Cygni-, U Geminorum- und ähnliche Veränderlichengruppen hinzuweisen: Das leider sehr spärliche spektroskopische Material widerspricht zumindest nicht der Annahme ihrer Verwandtschaft. Soweit mehrfache Ausbrüche beobachtet sind, scheinen sie auch, wie $\mathrm{Kukarkin}$ und

${ }^{4}$ K. H i m pe l, Z. Naturforschg. 1, 414 [1946]. 
$\mathrm{P}$ a r e $\mathrm{n}$ a $\mathrm{g} 0^{5}$ zuerst bemerkt haben, der gleichen Zyklus-Amplituden-Beziehung unterworfen zu sein wie die Novae. Mit wachsender Amplitude des Ausbruchs nimmt die Länge der dazwischenliegenden Zeitintervalle zu. Die auf Grund der Spektren zu erwartende hohe Temperatur, der kleine Farbenindex und die von $\mathrm{Kukarkin}$ und $\mathrm{Parenag}{ }^{6}{ }^{6}$ wahrscheinlich gemachte geringe Leuchtkraft $\left(+10^{m}\right)$ sprechen dafür, daß es sich hierbei vielleicht auch um eine Art weißer Zwerge handelt.

Die Wiederholungstendenz muß in einer Theorie des Novaphänomens einen Platz finden. Nach der Beziehung von $\mathrm{Kukarkin}$ und $\mathrm{Pare}$ $n$ a g o besteht sie für alle Novae und novaähnlichen Sterne, nur sind die z.T. zu erwartenden Zwischenzeiten so groß, daß noch keine Gelegenheit bestand, den zweiten Ausbruch zu beobachten.

Die Novae befinden sich im Russell-Diagramm in dem Gebiet zwischen der Hauptreihe und den weißen Zwergen. Das Durchlaufen der Hauptreihe wird mit dem Verbrauch des Wasserstoffs durch einen sehr stark temperaturabhängigen Kernprozeß, der für die Energieerzeugung verantwortlich ist und sie über $10^{10}-10^{11} \mathrm{Jahre}$ deckt, erklärt. Die letzte Phase vor dem Aufbrauchen des Wasserstoffs, wenn der Stern die Hauptreihe verläßt, wird rascher durchschritten als die vorhergehenden.

Die Existenz eines Resonanzeffektes der Kernreaktionen in bezug auf ihre Abhängigkeit von der Temperatur verlangt ein gegenüber dem Punktquellenmodell etwas modifiziertes Modell des Sternaufbaus. Die Energieproduktion steigt sehr rasch mit wachsender Temperatur zu einem Maximalwert an und fällt dann mit weiter steigender Temperatur wieder ab. Die Maxwell-Verteilung der thermischen Geschwindigkeiten verbreitert die Resonanzstelle etwas. Ga mow und Telle $\mathrm{r}^{7}$ haben gezeigt, daß der Anstieg zum Resonanzmaximum sehr rasch erfolgt, der $\mathrm{Ab}$ stieg mit steigender Temperatur jedoch sehr viel langsamer verläuft. Es ist also zu erwarten, daß bei Auftreten von Resonanz die Energieerzeugung in einem Stern im allgemeinen nicht im Mittelpunkt erfolgt, sondern überwiegend in einer

5 P. P a r e nag o u. B. K u k a r k i n, Veränderliche Sterne (Gorki) 4, 251 [1934].

${ }_{6}$ P. Paren a g o u. B. K u karkin, Veränderliche Sterne (Gorki) 4, 249 [1934].
Kugelschale stattfindet, deren Lage durch die Resonanzgeschwindigkeit der beteiligten Partikel, also die Temperatur der Schale, bestimmt wird.

Die wirkliche Verteilung der Energiequellen wird sich aber infolge des geschilderten Verlaufs der Resonanzkurve nicht nur in der Kugelschale abspielen, sondern sich auch noch gegen das Zentrum hin erstrecken. Die Energieerzeugung wird bei den in Betracht kommenden Temperaturen im allgemeinen auch noch im Mittelpunkt einen merklichen Betrag erreichen, da die Mittelpunktstemperaturen der Sterne nicht zu so hohen Teilchengeschwindigkeiten führen, üm Kernprozesse zum Stillstand zu bringen.

Wir führen nun folgende Hypothese ein: Sterne, die eine Inklination zu Novaausbrüchen aufweisen, besitzen einen sehr dichten entarteten Kern. Da innerhalb dieses Kerns die Temperatur nicht mehr nennenswert vom Mittelpunktsabstand abhängt, wird ihre Energie im wesentlichen in der Kugel entarteter Materie erzeugt werden. Die Temperatur ist sicher $>10^{7}$, also ausreichend, um Kernreaktionen in Gang $\mathrm{zu}$ halten. Masse ( Sonnenmasse), kleiner Radius und kleine Leuchtkraft der Praenova lassen das Vorhandensein eines entarteten Kerns nicht unwahrscheinlich erscheinen. Die Energieerzeugung ist infolge des Verbrauchs des im Kern vorhanden gewesenen Wasserstoffs am Erlöschen. Im Augenblick des Aufhörens bricht der Sternkern zusammen. Die äußeren Teile kontrahieren sehr rasch. Die am bisherigen Kern anliegenden Schichten entarten. Die damit verbundene Abnahme der Opazität befreit einen großen Betrag aufgespeicherter Energie, der nach außen abfließt, zu einer plötzlichen Erhöhung der Ausstrahlung führt und die äußersten Sternschichten als Nebelhülle forttreibt. Die nach innen auf den zusammengebrochenen Kern nachdringenden Schichten erreichen infolge Kontraktion die für Kernprozesse erforderliche Temperatur, so daß wieder Energieproduktion einsetzt. Da nur ein kleiner Bruchteil der Sternmasse in den nachstürzenden und neu entarteten Schichten und der Nebelhülle steckt, wird die Exnova von der gleichen Größenordnung und Leuchtkraft sein wie die Praenova, eher etwas heller, da die Kontraktion bis zur Wiederherstellung eines Gleichgewichtszustandes anhält. Der Abstieg nach einem Novaausbruch verläuft tatsächlich

7 G. Ga mow u. E. Telle r, Physic. Rev. 53. 608 [1938]. 
auch ganz erheblich langsamer als der Anstieg. Die nach innen kontrahierten Schichten erzeugen von neuem Energie, da sie den für die Kernreaktionen notwendigen Wasserstoff oder einen etwa sonst in der Kernzone ausgegangenen Reaktionsteilnehmer noch enthalten. Die Herstellung des Gleichgewichtes spiegelt sich in den sekundären Lichtschwankungen der Novae nach dem Ausbruch bzw. zwischen den Ausbrüchen wider. Ein stetiger Austausch von Sternmaterie und die damit verbundene Aufrechterhaltung der Ergiebigkeit subatomarer Energiequellen ist in merklichem Umfang nicht möglich, da die hierzu erforderliche Konvektion bei der sehr hohen Dichte des entarteten Kerns wohl kaum einsetzen kann.

Der Wiederholung des Novaausbruchs entspricht in dieser Vorstellung ein stufenweises Nachrücken immer weiter außen liegender Schich- ten auf den Kern. Der verhältnismäßig geringe Energieverlust bei einem Ausbruch erfordert, daß nur ein geringer Bruchteil der Sternmaterie jeweils an dem Prozeß teilnimmt. In diesem Sinne würde auch die erwähnte Zyklus-AmplitudenBeziehung verständlich. In bezug auf die zyklischen, novaähnlichen Veränderlichen wäre sie dahin zu deuten, daß bei ihnen der Vorgang infolge des noch kleineren Energieverlustes und des entsprechend verkleinerten Bruchteils beteiligter Sternmaterie noch sehr viel häufiger ablaufen muß als bei den eigentlichen Novae, bevor der Stern schließlich merkbar veränderte Eigenschaften, etwa Konstanz seines Lichtes, annimmt. Das sukzessive Fortschreiten der Entartung wird endlich zur Verminderung der Leuchtkraft des Sterns führen, bis er sich zu einem echten weißen Zwerg entwickelt hat.

\title{
Über eine Methode zur Herstellung von Intensitätsmarken bei der photographischen Photometrie
}

\author{
Von Hans-Karl Paetzold \\ Aus der Forschungsstelle für Physik der Stratosphäre, Weißenau, Kreis Ravensburg
}

(Z. Naturforschg. 2 a, 219-226 [1947]; eingegangen am 8. August 1946)

\begin{abstract}
Zur Aufnahme der Schwärzungskurve wird auf die photographische Platte das Beugungsbild eines Spaltes entworfen und dessen (berechnete) Intensitätsverteilung der Eichung zugrunde gelegt. Die Fehlereinflüsse dieser besonders für das kurzwellige Ultraviolett bestimmten Methode werden theoretisch diskutiert und experimentell geprüft. Als Anwendungsbeispiel wird die Eichung eines Platin-Stufenfilters durchgeführt.
\end{abstract}

$\mathrm{U}$ $\mathrm{m}$ die photographische Platte für photometrische $\mathrm{Z}$ wecke verwenden zu können, muß man in jedem einzelnen Falle eine Skala bekannter Intensitätsstufen auf die Platten aufdrucken, da die Zuordnung von Schwärzung und Intensität in komplizierter Weise von der Belichtung und Entwicklung, von der verwendeten Emulsion sowie von der Wellenlänge des Lichtes usw. abhängt. Zur Herstellung solcher Intensitätsmarken sind verschiedene Verfahren gebräuchlich, wie der $\mathrm{Ab}$ sorptionskeil, die Stufenblende nach $\mathrm{H}$ a n sen, Abschwächung des Lichtes nach dem 1/ $r^{2}$-Gesetz und anderes. Die Absorptionsmethoden leiden unter dem Nachteil, daß es keinen vollkommen nichtselektiven Absorber gibt. Die Anwendung des $1 / r^{2}$-Gesetzes stößt oft auf äußere Schwierigkeiten, wie zeitliche Inkonstanz der Lichtquelle, große Abmessungen u. ä. Diese Nachteile werden besonders störend im Gebiet des kurzwelligen Ultravioletts. Unterhalb von etwa 1200 A scheiden alle Methoden überhaupt aus, die Linsen oder Filter benötigen.

Für die Ultraviolettarbeiten der Forschungsstelle wurde ein Verfahren entwickelt, das einfach und übersichtlich anzuwenden und prinzipiell für jeden Spektralbereich brauchbar ist. Es beruht auf den Intensitätsverhältnissen bei der Beugung des Lichtes an einem Spalt ${ }^{1}$.

1 Siehe auch Ornstein-Burger-M oll, Objektive Spektralphotometrie [1932], S.94. 